Review

\title{
Coupled production and emission of short chain perfluoroalkyl acids from a fast developing fluorochemical industry: Evidence from yearly and seasonal monitoring in Daling River Basin, China ${ }^{\text {i⿱ }}$
}

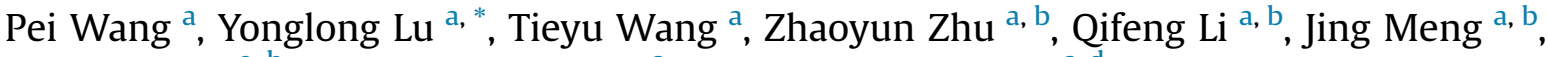 \\ Hongqiao Su ${ }^{\text {a, b}}$, Andrew C. Johnson ' ${ }^{\text {, Andrew J. Sweetman }}{ }^{\text {c, d }}$ \\ a State Key Lab of Urban and Regional Ecology, Research Center for Eco-Environmental Sciences, Chinese Academy of Sciences, Beijing 100085, China \\ ${ }^{\mathrm{b}}$ University of Chinese Academy of Sciences, Beijing 100049, China \\ ${ }^{\mathrm{c}}$ Centre for Ecology \& Hydrology, Maclean Building, Crowmarsh Gifford Wallingford, Oxon OX 10 8BB, UK \\ ${ }^{\mathrm{d}}$ Lancaster Environment Centre, Lancaster University, Lancaster LA1 4YQ UK
}

\section{A R T I C L E I N F O}

\section{Article history:}

Received 25 May 2016

Received in revised form

22 August 2016

Accepted 28 August 2016

Available online 31 August 2016

\section{Keywords:}

Yearly trend

Seasonal trend

PFBS

PFBA

PFOA

PFAA production

\begin{abstract}
A B S T R A C T
Short chain perfluoroalkyl acids (PFAAs) have been developed since 2002 by the major manufacturers to replace the conventional C8 and higher homologues, with much of the world production shifted to China in recent years. In this study, we conducted a continuous monitoring program over the period 2011 -2014 with seasonal monitoring in 2013 for PFAAs emitted from two rapidly developing fluorochemical industry parks located in the Daling River Basin, Northern China. The trend of PFAA contamination was identified, dominated by perfluorobutane sulfonic acid (PFBS), perfluorobutanoic acid (PFBA) and perfluorooctanoic acid (PFOA), with the maximum concentrations of $3.78 \mu \mathrm{g} / \mathrm{L}, 3.70 \mu \mathrm{g} / \mathrm{L}$, and $1.95 \mu \mathrm{g} / \mathrm{L}$, respectively. Seasonal monitoring uncovered the occasional emission of perfluorooctane sulfonic acid (PFOS). Construction trends of new facilities and associated manufacturing capacity of the main products were also analyzed to assess correlations with PFAA emissions. An assessment of the data over the period 2011-2014 found a positive correlation with fluorocarbon alcohol (FCA) production and emission of PFAAs. Groundwater and tap water around the main source indicated that the dominant PFAAs had different diffusion behaviors. PFBS levels were higher in surface water, while PFBA was dominant in groundwater and tap water, with PFOA levels being higher in downstream groundwater. Considering the continuous expansion and development of fluorochemical industry in the Daling River Basin, this study will provide abundant information on the effectiveness of risk assessment and management.
\end{abstract}

(C) 2016 Elsevier Ltd. All rights reserved.

\section{Introduction}

Perfluoroalkyl acids (PFAAs) have been widely used in both polymer applications, based on their water- and grease-repellent properties, and in surfactant applications, due to their unparalleled aqueous surface tension-lowering property since the 1950s (Buck et al., 2011). Historically, PFAAs were mostly produced and used in the long-chain forms, especially perfluorooctane sulfonic acid (PFOS) and perfluorooctanoic acid (PFOA) (Paul et al., 2009). However, the persistent, bio-accumulative and toxic (PBT)

\footnotetext{
This paper has been recommended for acceptance by Charles Wong.

* Corresponding author.

E-mail address: yllu@rcees.ac.cn (Y. Lu).
}

properties of long-chain PFAAs have led to their phase-out in production by the major manufacturers since 2000 (3M, 2000), followed by more and stricter regulations on their production, emission and use in multiple countries and regions including United States (US), European Union (EU), Norway, Canada and Germany (Holt, 2011). In 2009, PFOS and its related substances were listed in Annex B of the Stockholm Convention on Persistent Organic Pollutants (UNEP, 2009), which put the restrictions on a global scale. Apart from the major manufacturers, downstream users and individual customers are moving away from long-chain PFAAs due to health concerns. As a result, the perfluorinated chemical industry has been working on the development of alternatives (Wang et al., 2015b).

In 2002, the major manufacturer 3M developed new fluorochemical surfactants based on perfluorobutane sulfonic acid 
(PFBS). With four perfluorinated carbon atoms, PFBS was considered as a sustainable alternative to PFOS, and was claimed not to be PBT by $3 \mathrm{M}$ at that time. PFBS-based surfactants were subsequently commercialized in the US and other countries including China (3M, 2002). With four carbon atoms (three perfluorinated ones), perfluorobutanoic acid (PFBA) had also been manufactured for many years before 1998, particularly in making photographic film. $3 \mathrm{M}$ ceased the production of PFBA in 1998 due to decreased demand (3M, 2008). However, when C8 PFOA production was partially controlled in 2000, it appears that C4 PFBA manufacture recommenced. This was linked to very high levels of PFBA in the surrounding water environment of 3M's production site, where PFBA levels were dozens or even a hundred times greater than that for PFOA (Oliaei et al., 2013). Known fluorinated alternatives including short chain PFAAs and perfluoroether carboxylic and sulfonic acids (PFECAs and PFESAs) can adequately replace most C8 and higher homologue uses in many fluoropolymer applications. Although they are less persistent in the biota than the long chain PFASs, they are of concern because they do not break down in the environment and may cause similar toxicological effects as the long chain PFASs (Wang et al., 2013; Scheringer et al., 2014; Blum et al., 2015; Strynar et al., 2015). However, the consequences of the increased production and emission of short chain PFAAs are still largely unknown.

Currently, research on the sources of PFAAs has mostly focused on sewage treatment plants, atmospheric deposition, aqueous firefighting foams used at military bases, airports, and fire training facilities, as well as other non-fluorochemical sources in those countries or regions with strict regulation on PFAAs production (Post et al., 2012; Ahrens and Bundschuh, 2014). However, in China the most important source of PFAAs comes directly from fluoropolymer/-telomer manufacture due to the production shift in recent years. With the implementation of the Stockholm Convention, the production and emission of PFOS has been regulated in China, but other PFAAs remain outside of any restriction (POPS Action in China, 2014). Our previous studies identified the source and transportation routes of high levels of short chain PFAAs emitted from two fluorochemical industry parks in the Daling River Basin of North China (Wang et al., 2015a). However, the two parks have expanded very rapidly with frequent changes in production capacity of various fluorochemicals, so it is very important to trace the dynamic correlations between production and emission of dominant PFAAs.

A 4-year (2011-2014) and 4-season (2013) continuous monitoring of PFAAs in the Daling River was carried out in this study, and when combined with previous studies (Bao et al., 2010; Wang et al., 2011), a temporal trend of 7 years from 2008 to 2014 can be established, reflecting the fast development period of short-chain PFAAs in China. Furthermore, groundwater around Park 1 has also been analyzed to explore the different transport behavior of dominant PFAAs compared with surface water. The overall aim of the study was to establish the emission characteristics and temporal trends of PFAAs from an emerging fluorochemical industry in China, which can be used to provide a basis for effective surface water and groundwater management and risk assessment.

\section{Materials and methods}

\subsection{Sampling campaign}

\subsubsection{Yearly and seasonal sampling of river water in Daling River Basin}

As the two fluorochemical industry parks are located in the Xihe River, suitable sampling sites were identified upstream and downstream of the parks on this river up to the confluence point to the Daling River (site 1 to $8 \mathrm{X}$ ) and then along the Daling River down to the estuary (site 9 to 14 ) (Fig. 1). The distance from site 1 to site 8 is $82 \mathrm{~km}$, and from site 8 to site 14 is $90 \mathrm{~km}$. Sites on the Daling River further upstream of the confluence point with the Xihe River (site 15 to 18 and site 8D) were selected as control sites. According to our previous study in 2011, the two parks did not discharge sewage into the small tributaries of the Xihe River (Wang et al., 2015a), so these tributaries were not included in this study.

Yearly sampling campaigns were conducted in October of the years from 2011 to 2014, while seasonal samplings were conducted in January (winter), April (spring), July (summer) and October (autumn) in the year 2013. For all field samplings, only surface water (top 1-20 cm) was collected using $1 \mathrm{~L}$ pre-rinsed polypropylene (PP) bottles. Parameters including water temperature, $\mathrm{pH}$, dissolved oxygen, and conductivity were measured in situ using a HQd Portable and Benchtop Meter Configurator (HACH Company, USA) (Table S2-S3). Suspended substances in water samples were removed by settling for $24 \mathrm{~h}$ at room temperature and the supernatant was used for the following analysis.

\subsubsection{Ground water and tap water sampling in villages around Park 1}

The results of yearly (prior to 2014) and seasonal monitoring revealed that Park 1 had always been a significant point source of PFBS, PFBA and PFOA. So in 2014, along with the yearly sampling campaign in October, groundwater and tap water samples were also taken. Four villages in four directions local to Park 1 were selected (Fig. S1), batches of samples including ground water (5-7 m household wells) and tap water were sampled at randomly selected homes in each village using $1 \mathrm{~L}$ pre-rinsed PP bottles. The treatment of these samples was the same as that for the river water samples.

\subsection{Extraction and analysis of target analytes}

During the first sampling event in 2011, 17 PFAAs were analyzed and results indicated that perfluoroalkyl carboxylic acids (PFCAs) C13 and higher, and perfluoroalkane sulfonic acids (PFSAs) C10 were close to detection limits (Wang et al., 2015a). So in the later analysis, only 12 linear PFAAs including C4 to C12 PFCAs and 3 PFSAs were quantified (Table S4). A $400 \mathrm{~mL}$ aliquot of water spiked with $10 \mathrm{ng}$ mass-labelled standards was extracted using OASIS WAX-SPE following published methods (Taniyasu et al., 2005) with some modifications. Briefly, prior to loading samples, the Oasis WAX cartridges ( $6 \mathrm{cc}, 150 \mathrm{mg}, 30 \mu \mathrm{m}$, Waters, Milford, MA) were preconditioned with $4 \mathrm{~mL}$ of $0.1 \% \mathrm{NH}_{4} \mathrm{OH}$ in methanol, $4 \mathrm{~mL}$ of methanol, and $4 \mathrm{~mL}$ of Milli-Q water. After loading the samples, cartridges were washed with $4 \mathrm{~mL} 25 \mathrm{mM}$ ammonium acetate $(\mathrm{pH}=4)$ and air-dried. Target analytes were then eluted with $4 \mathrm{~mL}$ of methanol and $4 \mathrm{~mL}$ of $0.1 \% \mathrm{NH}_{4} \mathrm{OH}$ in methanol, respectively. The extracts were reduced to $1 \mathrm{~mL}$ under high purity nitrogen (99.999\%) and passed through a nylon filter ( $13 \mathrm{~mm}, 0.2 \mu \mathrm{m}$, Chromspec, Ontario, Canada), then transferred into a $1.5 \mathrm{~mL}$ PP snap top brown glass vial with polyethylene (PE) septa. Individual PFAA was separated and quantified via Agilent 1290 Infinity HPLC System coupled to an Agilent 6460 Triple Quadrupole LC/MS System (Agilent Technologies, Palo Alto, CA) operated in the negative electrospray ionization (ESI-) mode. Conditions under which the instrument was operated were listed in Table S5.

\section{3. $Q A / Q C$}

Since the manufacturers and local residents were not informed of the sampling campaign, this study can be assumed to reflect routine production activities in the two parks. The yearly/seasonal sampling was conducted at the same date of the year/season and 


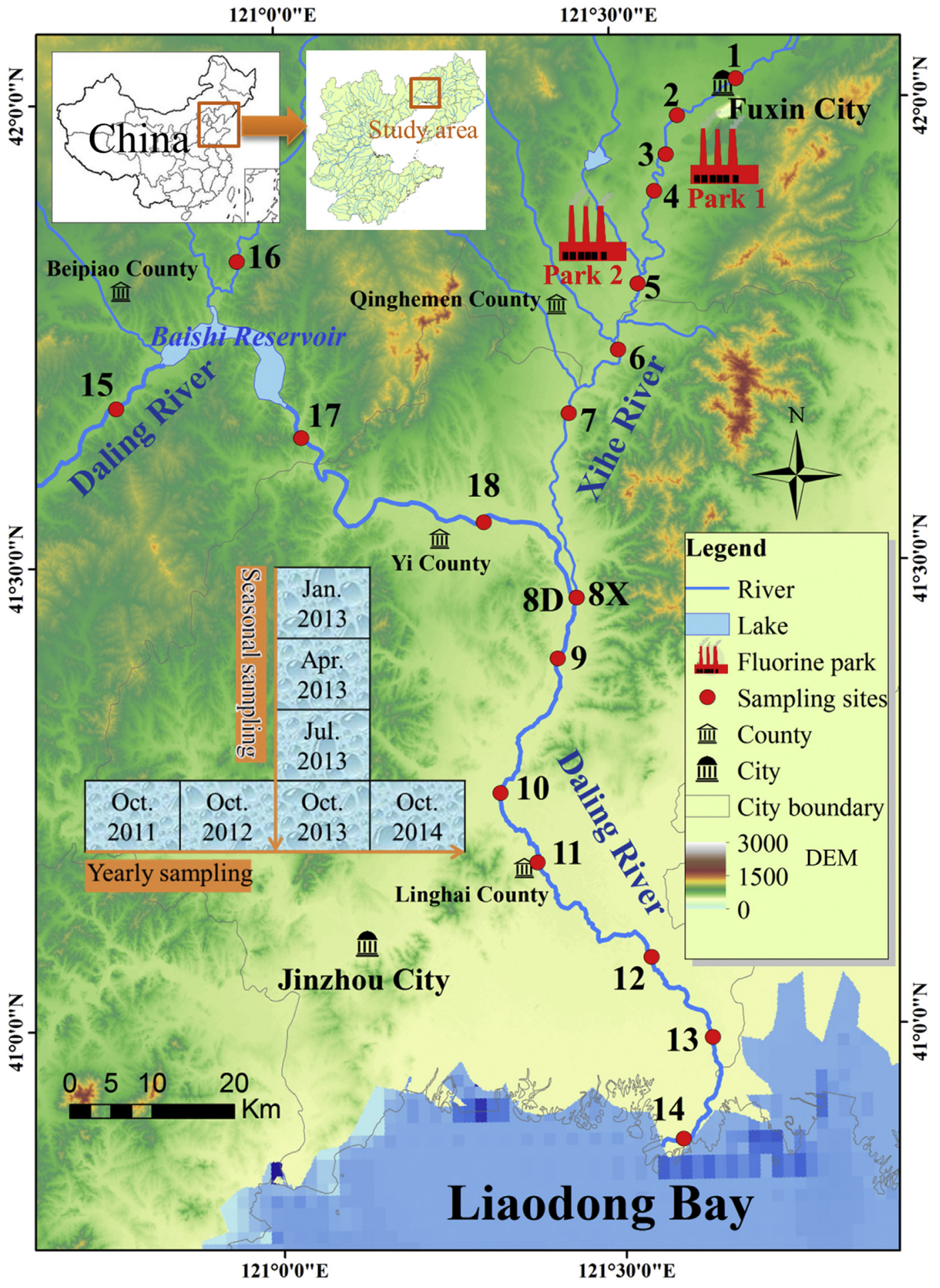

Fig. 1. Sampling sites in the Daling River Basin.

the same time of a day as much as possible. These were designed to provide parallel comparisons. Use of polytetrafluoro-ethylene (PTFE) or other fluoro-polymer/-telomer materials was avoided throughout the study. In order to avoid cross contamination during sampling, the outside of the $1 \mathrm{~L}$ PP bottles was immediately washed with pure water after the samples were taken, wiped with clean towel paper and then kept in sealed PP bags. Field, transport, procedure and solvent blanks were prepared with every sample batch to monitor potential interferences. Quantification of the 12 PFAAs in water were carried out using 10-point external standard curves with concentrations ranging from 0.01 to $1000 \mathrm{ng} / \mathrm{mL} .10 \mathrm{ng} /$ $\mathrm{mL}$ of mass-labelled standards as surrogates were also added to the samples to monitor the matrix effect. The injection volume was $5 \mu \mathrm{L}$. The regression coefficients $\left(\mathrm{R}^{2}\right)$ for all the target analytes were over 0.99 . The limit of detection (LOD) and limit of quantification (LOQ) were defined as the peak of analyte that needed to yield a 
signal-to-noise $(\mathrm{S} / \mathrm{N})$ ratio of 3:1 and 10:1, respectively. LOD and LOQ in this study were checked along every sampling event, and the final values were combined and presented at the highest ones. LODs ranged from 0.01 to $0.15 \mathrm{ng} / \mathrm{L}$, while LOQs ranged from 0.06 to $0.71 \mathrm{ng} / \mathrm{L}$. Recovery tests were also conducted along all sampling events, and the values were combined together to give the means and standard deviations for individual PFAA. Matrix spike recoveries ranged from 73 to $113 \%$, while procedure recovery ranged from 81 to $124 \%$. For extracts containing concentrations of PFAAs greater than $1000 \mathrm{ng} / \mathrm{mL}$, the samples were reduced in volume and extracted again to make sure the concentrations of PFAAs were within the calibration range. Detailed QA/QC measurements of target PFAAs are given in the Supplementary Material.

\subsection{Statistical and spatial analysis}

Statistical analysis was performed using SPSS Statistics V20.0 (SPSS Inc. Quarry Bay, HK), Minitab 16 (Minitab Inc. PA, USA) and OriginPro 9.1 (Northampton, MA, USA). Prior to the statistical analysis, tests of normality were carried out to ensure that data met the assumptions used for further analysis. Trend analysis for dominant PFAAs was processed using one-way ANOVA with posthoc tukey test at the significance level of 0.05 and $95 \%$ Confidence. Spatial distributions of the sampling sites were illustrated using the Arcmap module in ArcGIS V10.0 software (ESRI, Redland, CA). GIS data used for sampling design was obtained from the National Geomatics Center of China (Haidian District, BJ).

\subsection{Data representation}

Due to the large variation in the orders of magnitude, and the fact that the accuracy of all data was presented with three significant figures, value units for concentrations in water were ng/L or $\mu \mathrm{g} / \mathrm{L}$.

\section{Results and discussion}

\subsection{Yearly trend of PFAAs emitted to the Daling River water}

From 2011 to 2014, PFBS and PFBA have always been the dominant PFAAs, followed by PFOA, and then C5-C7 PFCAs (Table 1, Table S6-S9, Fig. 2). The C9-C12 PFCAs and PFHxS were detected in both low ratios and low concentrations, indicating they were seldom produced in the study area, thus these PFAAs will not be discussed in the following sections. The highest concentrations of
PFBS were $2.90 \mu \mathrm{g} / \mathrm{L}$ in 2011, $2.34 \mu \mathrm{g} / \mathrm{L}$ in 2012, $2.71 \mu \mathrm{g} / \mathrm{L}$ in 2013 and $3.78 \mathrm{ng} / \mathrm{L}$ in 2014. For PFBA, the highest concentrations were $1.35 \mu \mathrm{g} / \mathrm{L}$ in $2011,1.57 \mu \mathrm{g} / \mathrm{L}$ in $2012,3.70 \mu \mathrm{g} / \mathrm{L}$ in 2013 and $2.58 \mu \mathrm{g} / \mathrm{L}$ in 2014. For PFOA, the highest concentrations were $348 \mathrm{ng} / \mathrm{L}$ in 2011, $675 \mathrm{ng} / \mathrm{L}$ in 2012, $1.95 \mu \mathrm{g} / \mathrm{L}$ in 2013 and $772 \mathrm{ng} / \mathrm{L}$ in 2014, respectively. One-way ANOVA analysis for PFAA concentrations at sites downstream of the parks in the Xihe River (site 3 to site $8 \mathrm{X}$ ) indicated that PFBA levels showed a significant increase from 2011 to 2014, PFBS levels showed a significant increase from 2012 to 2014, and PFOA levels showed no clear trend (Table S16). Concentrations of PFOS were far below those of the three dominant PFAAs, with the overall highest concentration of $12.6 \mathrm{ng} / \mathrm{L}$ observed in 2011. Among the C5-C7 PFCAs, C6 PFHxA was dominant with the highest concentration up to $795 \mathrm{ng} / \mathrm{L}$ in 2013. C6 PFAAs were also considered to be one of the potential alternatives to long chain PFAAs (Wang et al., 2015b). However, even in the site with the highest concentration, PFHxA level was a factor of 5 less than PFBA, indicating a lower importance than C4 PFBA as an alternative.

As to the spatial distribution, the highest concentrations of PFBS and PFBA were associated with Park 1 (site 3 and 4) throughout the four years (Fig. 2). For PFOA, the spike in concentration from site 3 was less obvious than those of PFBS and PFBA except in 2013. Further distribution from Park 2 maintained the elevated levels of the three dominant PFAAs from site 5 to site $8 X$, whilst the decreasing levels in site 6 might be caused by the dilution from the tributaries entering the Xihe River. Along the Daling River (from site $8 \mathrm{X}$ to site 12 ), concentrations were generally reduced by over $50 \%$ due to dilution. The fluctuation of concentrations in site 13 and site 14 at the estuary could be affected by factors including the frequent mixture of fresh and saline water, aquaculture pools using the river water, construction activities, and so forth. Meanwhile, concentrations of PFAAs at the reference sites from 15 to 17 indicated no other point sources from the upstream parts of the Daling River over the study period. The relatively notable PFAAs levels in site 18 and 8D could originate from domestic emissions in Yi County.

\subsection{Seasonal trend of PFAAs emitted into the Daling River water}

Seasonal monitoring in 2013 was conducted as a supplement for the yearly data, to illustrate the general scientific findings in more detail. Differences in PFAA levels could be observed over the different seasons of 2013 (Table 2, Table S10-S13, Fig. 3). For PFBS, the overall concentrations from site 1 to site 14 were highest in summer (max. $3.87 \mu \mathrm{g} / \mathrm{L}$ ) and autumn (max. $2.71 \mu \mathrm{g} / \mathrm{L}$ ) with lower levels in spring (max. $1.98 \mu \mathrm{g} / \mathrm{L}$ ) and winter (max. $690 \mathrm{ng} / \mathrm{L}$ ), while

Table 1

Summary of PFAAs concentrations in yearly monitoring (ng/L).

\begin{tabular}{|c|c|c|c|c|c|c|c|c|}
\hline Time & & PFBA & PFPeA & PFHxA & PFHpA & PFOA & PFBS & PFOS \\
\hline \multicolumn{9}{|c|}{ Yearly } \\
\hline \multirow[t]{4}{*}{2011} & Min. & 0.31 & 0.04 & 0.03 & 0.04 & 0.09 & 0.04 & 0.05 \\
\hline & Max. & 1350 & 82.2 & 59.2 & 15.9 & 348 & 2900 & 12.6 \\
\hline & Mean & 374 & 20.4 & 18.3 & 4.24 & 132 & 856 & 3.97 \\
\hline & Median & 223 & 15.9 & 14.3 & 3.34 & 102 & 386 & 2.50 \\
\hline \multirow[t]{4}{*}{2012} & Min. & 1.56 & 0.25 & 0.09 & 0.08 & 0.58 & 0.47 & 0.16 \\
\hline & Max. & 1570 & 81.2 & 180 & 45.5 & 675 & 2340 & 2.27 \\
\hline & Mean & 276 & 19.6 & 49.4 & 12.7 & 200 & 516 & 0.89 \\
\hline & Median & 156 & 12.1 & 29.5 & 7.80 & 113 & 253 & 0.77 \\
\hline \multirow[t]{4}{*}{2013} & Min. & 1.62 & 0.05 & 0.08 & 0.10 & 0.61 & 0.74 & 0.06 \\
\hline & Max. & 3700 & 198 & 795 & 103 & 3950 & 2710 & 11.4 \\
\hline & Mean & 1050 & 41.8 & 100 & 22.3 & 533 & 830 & 3.59 \\
\hline & Median & 945 & 34.0 & 56.4 & 20.1 & 344 & 712 & 2.43 \\
\hline \multirow[t]{4}{*}{2014} & Min. & 1.42 & ND & 0.11 & 0.10 & 2.17 & ND & 0.47 \\
\hline & Max. & 2580 & 56.1 & 196 & 55.0 & 772 & 3780 & 6.95 \\
\hline & Mean & 830 & 23.6 & 60.7 & 19.3 & 239 & 1090 & 2.60 \\
\hline & Median & 536 & 21.1 & 42,2 & 15.5 & 155 & 668 & 1.52 \\
\hline
\end{tabular}



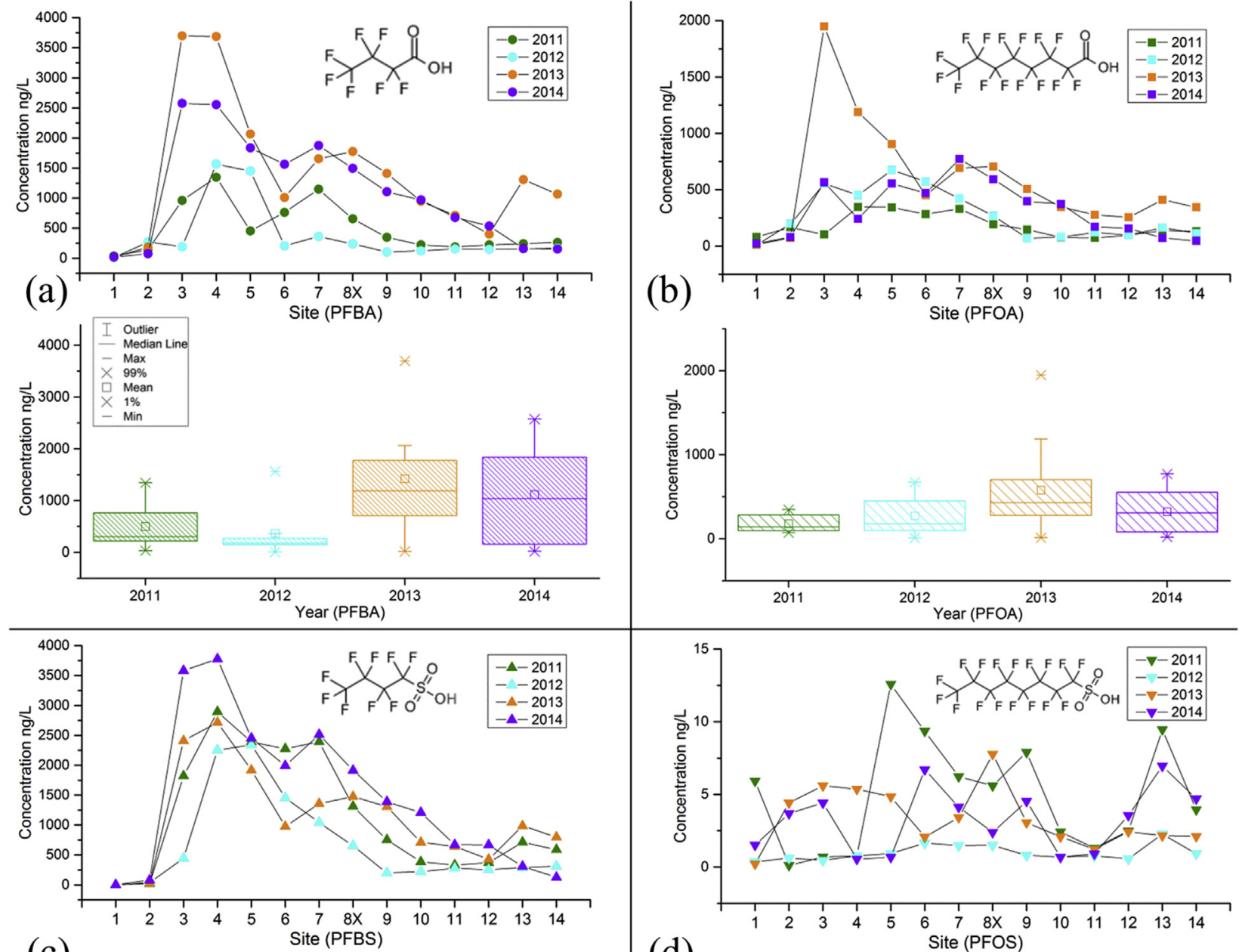

(c)

(d)
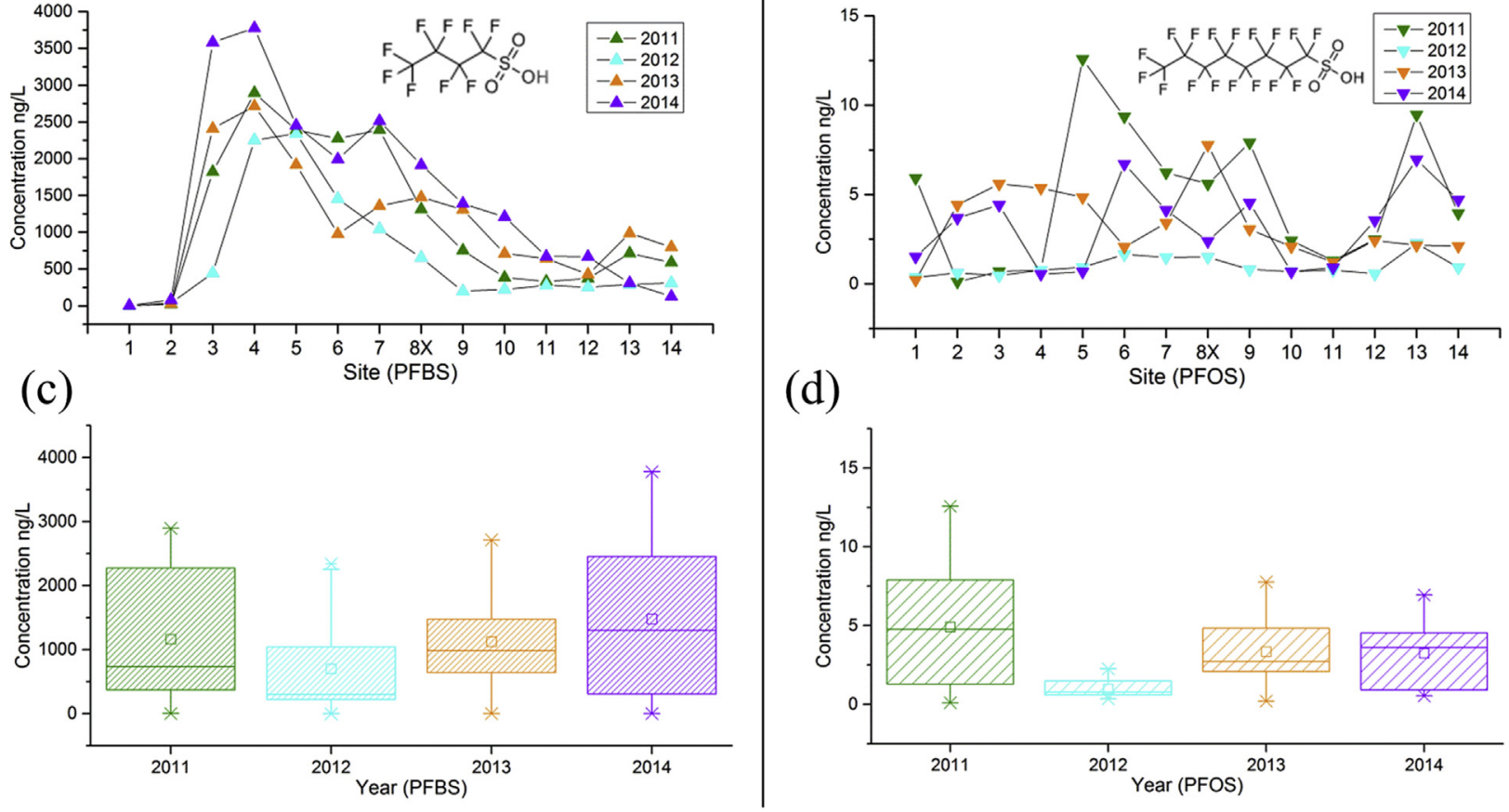

Fig. 2. Temporal trends of main PFAAs in the Xihe and Daling River water from 2011 to 2014.

the trend was autumn $(3.70 \mu \mathrm{g} / \mathrm{L})>$ summer $(2.44 \mu \mathrm{g} / \mathrm{L})>$ spring $(1.97 \mu \mathrm{g} / \mathrm{L})>$ winter $(628 \mathrm{ng} / \mathrm{L})$ for PFBA, and summer $(2.28 \mu \mathrm{g} /$ L) $>$ autumn $(1.95 \mu \mathrm{g} / \mathrm{L})>$ winter $(753 \mathrm{ng} / \mathrm{L})>\operatorname{spring}(749 \mathrm{ng} / \mathrm{L})$ for PFOA, respectively. PFOS was found at low concentrations, but there was an apparent point-source emission in site 5 , downstream of Park 2, with a concentration of $483 \mathrm{ng} / \mathrm{L}$. Relatively high levels of C6 PFHxA have been emitted since the summer of 2013. This indicated that even though PFBS, PFBA and PFOA were the dominant PFAAs emitted from the two parks, other homologues including PFOS could still be produced and emitted in some occasions. This was maybe due to the unstable status of construction and production in the parks, which will be discussed later.

For the important compounds of PFBA, PFBS and PFOA, the highest levels in river water were found in either summer or autumn. Given that rainfall and hence river flow (and dilution) tends to be highest in summer and autumn in China, this may appear as something of a surprise. Considering the occasional high emission of PFOS (483 ng/L) in spring, the seasonal pattern of PFAA emissions might depend on fluctuating request from market. Besides, the construction of existing and new facilities, and seasonal variations in river discharge would also have influences on the emission patterns. All the factors could form general industrial cycles, which were also partially reflected in the trend of yearly sampling. There were several studies on the seasonal variations of PFAAs in water environment (Tsuda et al., 2010; Zhao et al., 2015), while the overall levels were low without identification of potential sources. As far as we know, this is the first study on yearly and seasonal monitoring of PFAAs emitted from fluorochemical 
Table 2

Summary of PFAAs concentrations in seasonal monitoring (ng/L).

\begin{tabular}{|c|c|c|c|c|c|c|c|c|}
\hline Time & & PFBA & PFPeA & PFHxA & PFHpA & PFOA & PFBS & PFOS \\
\hline \multicolumn{9}{|c|}{ Seasonal (2013) } \\
\hline \multirow[t]{4}{*}{ Winter } & Min. & 0.64 & 0.06 & 0.33 & 0.12 & 0.27 & 0.04 & 0.87 \\
\hline & Max. & 628 & 139 & 183 & 29.4 & 753 & 690 & 39.9 \\
\hline & Mean & 231 & 38.7 & 45.0 & 8.20 & 202 & 209 & 7.94 \\
\hline & Median & 171 & 12.9 & 17.0 & 5.69 & 102 & 130 & 4.08 \\
\hline \multirow[t]{4}{*}{ Spring } & Min. & 1.36 & 0.05 & 0.02 & 0.08 & 0.47 & 0.04 & 0.59 \\
\hline & Max. & 1970 & 79.6 & 168 & 36.8 & 749 & 1980 & 483 \\
\hline & Mean & 351 & 15.7 & 32.8 & 8.53 & 150 & 409 & 54.0 \\
\hline & Median & 125 & 5.06 & 9.65 & 2.84 & 47.9 & 149 & 6.54 \\
\hline \multirow[t]{4}{*}{ Summer } & Min. & 5.78 & 0.21 & 0.15 & 0.38 & 2.51 & 0.26 & 1.02 \\
\hline & Max. & 2440 & 273 & 603 & 119 & 2280 & 3870 & 21.2 \\
\hline & Mean & 643 & 60.1 & 122 & 26.8 & 495 & 969 & 4.27 \\
\hline & Median & 372 & 20.7 & 43.0 & 10.1 & 179 & 581 & 2.90 \\
\hline \multirow[t]{4}{*}{ Autumn } & Min. & 1.62 & 0.05 & 0.08 & 0.10 & 0.61 & 0.52 & 0.06 \\
\hline & Max. & 3700 & 198 & 795 & 103 & 3950 & 2710 & 11.4 \\
\hline & Mean & 1050 & 41.8 & 100 & 22.3 & 533 & 830 & 3.49 \\
\hline & Median & 945 & 34.0 & 56.4 & 20.1 & 344 & 712 & 2.43 \\
\hline
\end{tabular}

industry.

\subsection{PFAA emission characteristics related to construction and production}

The dominant PFAAs levels showed different trends over the study period (Fig. 4c), which can be used to identify potential changes to emission sources and can also reflect the dynamic of market need and production capacity. Concentration ratios can provide comparisons on the temporal variations of the dominant PFAAs. It can be seen from Table 3 that, the mean values of the PFBS/ PFBA ratio from site 3 to site $8 X$ were 2.7 and 3.0 in 2011 and 2012, respectively. But the ratios became 0.8 in 2013 and 1.4 in 2014. For PFBA/PFOA ratios, the mean values also showed a general increasing trend from 2012 to 2014. These might imply that emission of PFBA increased more than that of PFBS and PFOA over the most recent years, with PFBA more important in the fluorochemical applications. Spatial variations of the concentration ratios indicated that the emission intensity from the two parks for the dominant PFAAs had been changing. This was consistent with the status that the planning and construction of fluoropolymer facilities in the two parks had been changing all the time.

In our previous study in 2011, there were limited fluoropolymer facilities in the study area, and production processes could be identified (Wang et al., 2015a). However, due to continuing site development, this task has become more complex. From 2011 to 2014, there were dozens of facilities under development, planning to produce or planning to construct (Fig. 4a), especially the number (accumulated value) of facilities under production showed a steadily increasing trend. This brought an increasing capacity of organo-fluorine products that related to the emission of PFAAs (Fig. 4b, Table S15). Especially, the production of fluorocarbon alcohol (FCA) and PTFE would directly generate or use PFAAs (Fig. 5), but the trends were quite different (Fig. 4c). According to the manufacturers, FCA including perfluoroalkyl iodides (PFAIs), fluorotelomer alcohols (FTOHs) and related products are all featured products in the study area, so the production capacity showed an increasing trend. There are various processes including electrochemical fluorination and telomerization that could generate the emission of the dominant PFAAs (Fig. 5). The production of PTFE would use PFOA as a processing aid, and lead to the emission of PFOA (European Commission, 2010). However, PTFE and its related products have not been the main products for the fluorochemical manufacturers in this study, which have a sum capacity of only thousands of tons. So when other manufacturers with
PTFE as one of their main products increased the production capacity, such as one in the Xiaoqing River Basin in the same economic circle, with a PTFE capacity of 49,000 tons (Wang et al., 2016), the manufacturers in this study chose to reduce the PTFE capacity. Besides, there is a large and increasing capacity for the production of various intermediates for pesticides and medicines using organofluorine. Furthermore, the development of a central wastewater treatment plant (WWTP) in the parks could also have effects on the emission of PFAAs to the river, as some processes were designed specifically for the removal of fluorine in wastewater from individual fluorochemical facilities in Park 2, which needs more detailed study.

\subsection{PFAAs in groundwater and tap water around Park 1}

The yearly and seasonal monitoring data indicated that Park 1 discharged high levels of PFBS and PFBA directly and continuously to the Xihe River. It is possible that these short-chain PFAAs might migrate to the groundwater through river leakage or other pathways (Liu et al., 2016), and then lead to the exposure of local residents via water abstraction using shallow wells $(5-7 \mathrm{~m})$. Unlike short-chain PFAAs that are usually produced at liquid form, longchain PFAAs are present at solid or powder forms (Wang et al., 2015a), which make them more susceptible to atmospheric dispersion followed by migration into groundwater via precipitation (Davis et al., 2007). In this study, very high levels of PFAAs were detected in the groundwater taken from the wells in villages nearby Park 1 (Table 4, Table S14, Fig. 6). The site W1 is very close to site 3 in the Xihe River $(<100 \mathrm{~m})$, comparisons on PFAAs levels showed that unlike in river water, PFOA was the dominant compound here. In this case the PFOA was found at W1 with concentration up to $4.15 \mu \mathrm{g} / \mathrm{L}$ and an overall contribution of $37.5 \%$ of $\sum$ PFAAs (sum of PFAAs), followed by PFBA (3.61 $\mu \mathrm{g} / \mathrm{L}, 32.7 \%)>$ PFHxA $(1.18 \mu \mathrm{g} / \mathrm{L}$, 10.6\%) > PFPeA (951 ng/L, 8.6\%) > PFBS (588 ng/L, 5.3\%) > PFHpA (579 ng/L, 5.2\%). For W2-W4, which are located further away from Park 1, PFAAs levels decreased and contributions of individual PFAA also changed. PFBA was more dominant at the W2 groundwater site with concentration of $1.78 \mu \mathrm{g} / \mathrm{L}$ and contribution of $41.4 \%$, followed by PFOA $(1.39 \mu \mathrm{g} / \mathrm{L}, 32.4 \%)$. PFBS accounted for a larger potion (865 ng/L, 20.2\%). The concentrations and profiles of PFAAs were quite comparable in W3 and W4 with $\sum$ PFAAs of $536 \mathrm{ng} / \mathrm{L}$ and $463 \mathrm{ng} / \mathrm{L}$, respectively. PFBA accounted for about $80 \%$, contributions of PFOA reduced to $12.0 \%$ and $17.6 \%$, respectively, while PFBS and C5-C7 PFACs accounted for even less. The spatial distribution of W1-W4 indicated that apart from the distance to Park 1, local 

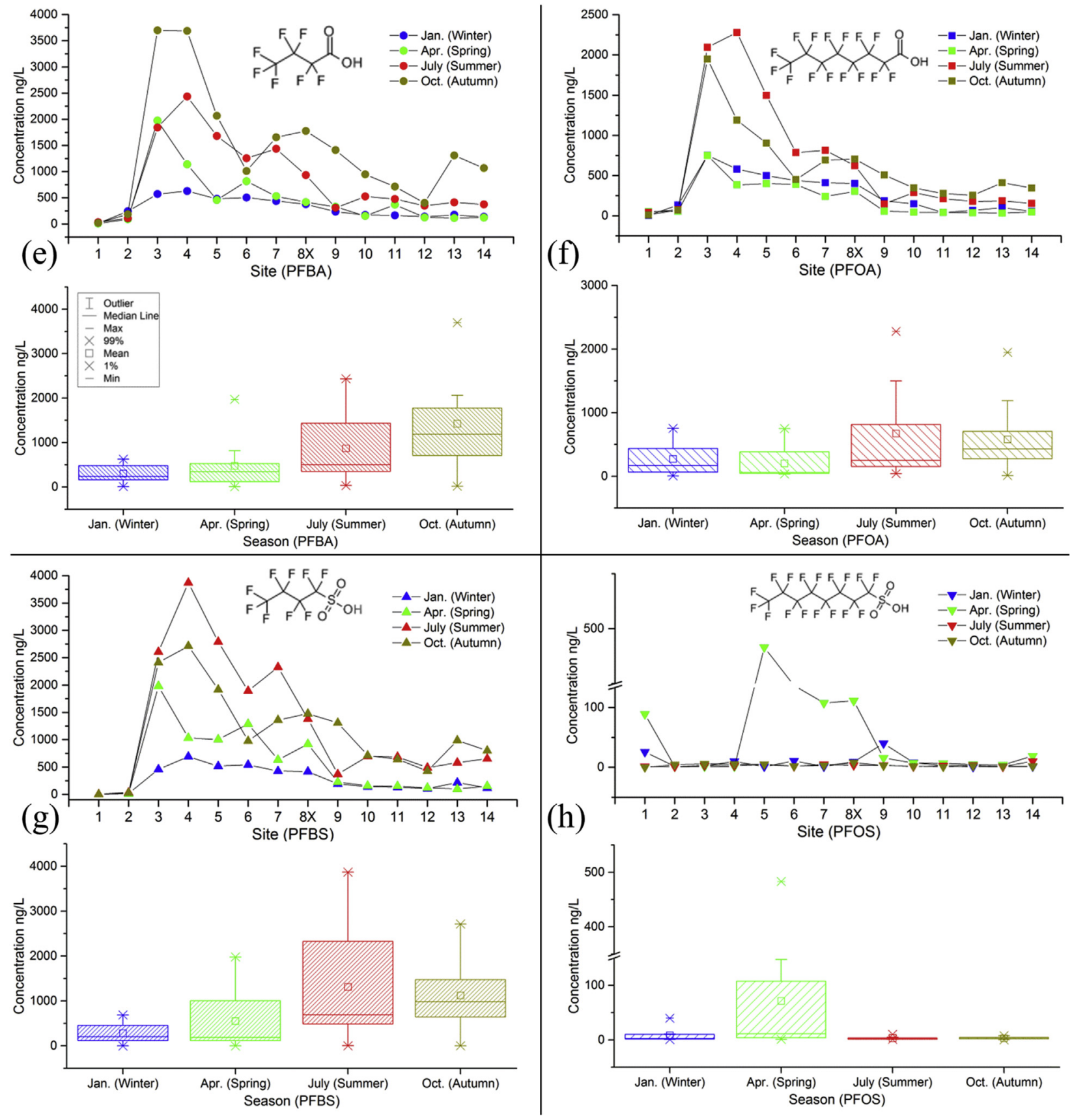

Fig. 3. Levels of main PFAAs in the Xihe and Daling River water in four seasons of the year 2013.

environmental conditions combined with PFAA properties may explain the varied levels and profiles of PFAAs in groundwater. W1 is adjacent to the Aliphatic fluoride zone of Park 1 (Fig. 4), which is the zone of direct production and use of PFOA. PFOA could be released as dust, solid waste or impurities in products, and migrate with runoff into groundwater. The high mobility of PFBA led to its high presence in W3 and W4, and dominance in W1 and W2 (Oliaei et al., 2013). PFBS was mainly emitted to and found in river water. $\sum$ PFAAs in tap water of W1-W3 were all below $10 \mathrm{ng} / \mathrm{L}$, only tap water in W4 presented $\sum$ PFAAs level of $74.2 \mathrm{ng} / \mathrm{L}$.

Previous studies reported that in areas with relatively high PFOA levels in surface water, the tap water could also contain higher PFOA levels (Saito et al., 2004). In this study, PFOA levels in tap water of site W4 exceeded the Preliminary Health-Based Guidance value (40 ng/L) in New Jersey, USA (NJDEP, 2007), and especially, the newest Vermont Drinking Water Health Advisories (20 ng/L) and USEPA Lifetime Health Advisories (20 ng/L), which were both derived in 2016 (HealthVermont, 2016; USEPA, 2016). According to the general office of the local tap water company, the source of tap water comes from several reservoirs nearby, which are protected well. However, there are five waterworks, so it is not clear why the tap water nearby Park 1 presented notable PFAAs levels, which requires immediate investigation by the supplier. As for the groundwater in domestic wells, the local residents have been aware of the pollution from the fluorochemical facilities, and kept it away from drinking for a long time. However, the groundwater is still used in daily life for irrigation of crops and vegetables, watering domestic animals, washing clothes, cleaning etc. Therefore, 

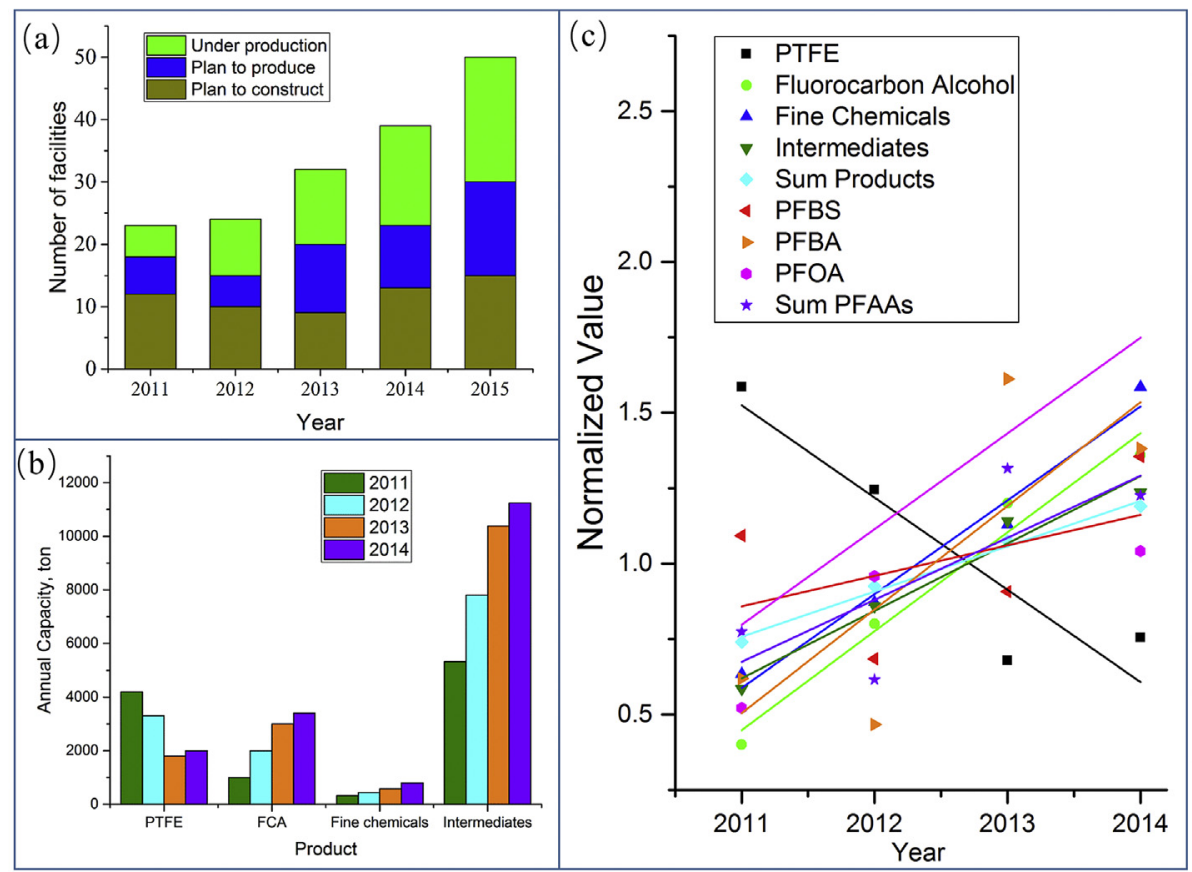

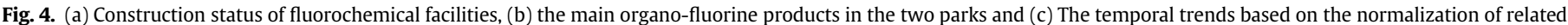
values divided by median.

Table 3

Concentration ratios of PFBS/PFBA and PFBA/PFOA in the sites 3-8X.

\begin{tabular}{|c|c|c|c|c|c|c|c|c|}
\hline \multirow[t]{2}{*}{ Site } & \multicolumn{4}{|c|}{ PFBS/PFBA } & \multicolumn{4}{|c|}{ PFBA/PFOA } \\
\hline & 2011 & 2012 & 2013 & 2014 & 2011 & 2012 & 2013 & 2014 \\
\hline 3 & 1.9 & 2.3 & 0.7 & 1.4 & 9.4 & 0.3 & 0.9 & 4.6 \\
\hline 4 & 2.2 & 1.4 & 0.7 & 1.5 & 3.9 & 3.5 & 3.1 & 10.6 \\
\hline 5 & 5.3 & 1.6 & 0.9 & 1.3 & 1.3 & 2.1 & 2.3 & 3.3 \\
\hline 6 & 3.0 & 7.0 & 1.0 & 1.3 & 2.7 & 0.4 & 2.2 & 3.3 \\
\hline 7 & 2.1 & 2.9 & 0.8 & 1.3 & 3.5 & 0.9 & 2.4 & 2.4 \\
\hline $8 \mathrm{X}$ & 2.0 & 2.7 & 0.8 & 1.3 & 3.4 & 0.9 & 2.5 & 2.5 \\
\hline Mean & 2.7 & 3.0 & 0.8 & 1.4 & 4.0 & 1.3 & 2.2 & 4.5 \\
\hline
\end{tabular}

potential adverse effects may still exist if groundwater from W1 and W2 was used, as PFOA levels in the wells of the two villages exceeded almost all criteria values (Table 4). Besides, there is a trend for more stringent safety values for PFOA (MDH, 2007; 2011; USEPA, 2014; 2016), and considering the long emission history of PFOA and the accumulation of PFOA in groundwater, further studies are needed to ensure that the development of the fluorochemical industry would do little harm to local residents.

\section{Conclusions and perspectives}

The yearly monitoring indicated that the emissions of PFAAs from two fast developing fluorochemical industry parks in North China were continuous with a general increasing trend from 2008 to 2014. PFBS, PFBA and PFOA were identified as the most dominant PFAAs, followed by C5-C7 PFCAs. Seasonal monitoring identified summer and autumn as the peak river contamination periods in this location, which also identified occasional PFOS emission. Construction of new facilities and annual capacity of the main organo-fluorine products had potential relationship with the dominant PFAAs emissions. The capacities of FCA showed an increasing trend along with most processes that generate the emissions of PFAAs. Analysis of groundwater and tap water samples taken from the nearby villages of Park 1 showed different profiles of PFAAs in groundwater compared to river water, with PFBA having

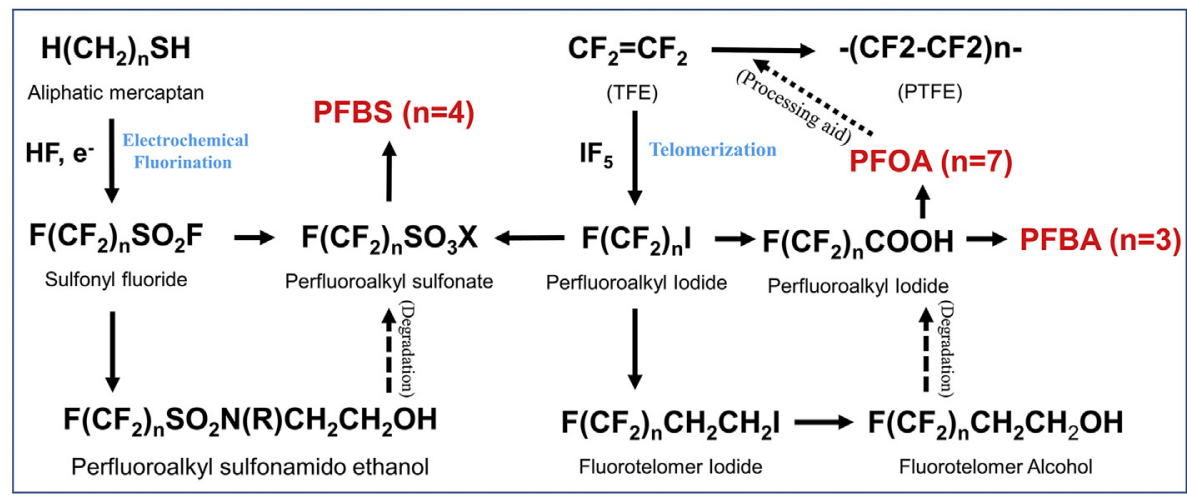

Fig. 5. Main production processes and products in the parks correlated with the emission of the dominant PFAAs. 
Table 4

Concentrations of the main PFAAs (ng/L) in the groundwater and tap water in this study, and available health drinking water criteria (ng/L) in the world.

\begin{tabular}{|c|c|c|c|c|c|c|}
\hline Region & Type & PFBA & PFOA & PFBS & PFOS & year \\
\hline \multicolumn{7}{|l|}{ This study } \\
\hline \multirow[t]{2}{*}{ W1 } & Groundwater & 3610 & 4150 & 588 & 1.05 & 2014 \\
\hline & Tap water & 4.68 & 2.38 & 1.09 & 0.30 & 2014 \\
\hline \multirow[t]{2}{*}{ W2 } & Groundwater & 1780 & 1390 & 865 & 0.20 & 2014 \\
\hline & Tap water & 4.34 & 1.74 & 0.93 & 0.35 & 2014 \\
\hline \multirow[t]{2}{*}{ W3 } & Groundwater & 434 & 64.5 & 22.7 & 0.20 & 2014 \\
\hline & Tap water & 5.59 & 1.21 & 1.64 & 0.43 & 2014 \\
\hline \multirow[t]{2}{*}{ W4 } & Groundwater & 352 & 81.7 & 22.4 & 0.15 & 2014 \\
\hline & Tap water & 92.3 & 74.2 & 97.8 & 0.13 & 2014 \\
\hline United States & Regulatory value & & & & & \\
\hline Vermont & Drinking Water Health Advisories & - & $20^{\mathrm{a}}$ & - & $20^{\mathrm{a}}$ & 2016 \\
\hline New Jersey & Preliminary Health-Based Guidance & - & 40 & - & - & 2007 \\
\hline USEPA & Lifetime Health Advisories & - & $70^{\mathrm{b}}$ & - & $70^{\mathrm{b}}$ & 2016 \\
\hline North Carolina & Interim Maximum Allowable Concentration & - & 1000 & - & - & 2010 \\
\hline Minnesota & Chronic Health Risk Limit & 7000 & 300 & 7000 & 300 & 2011 \\
\hline Maine & Maximum Exposure Guideline & - & 100 & - & - & 2014 \\
\hline \multirow[t]{3}{*}{ United Kingdom } & Further Provision & - & 300 & - & 300 & 2009 \\
\hline & Potential Danger to Human Health & - & 5000 & - & 1000 & \\
\hline & Notification of Events & - & 45,000 & - & 9000 & \\
\hline Germany & Health-Related Indication Value & 7000 & 300 & 3000 & 300 & $2006 / 2010$ \\
\hline Canada & Drinking Water Protection & - & 700 & - & 300 & 2010 \\
\hline Italy & $\mathrm{AA}-\mathrm{EQS}^{\mathrm{C}}$ (Freshwater) & 7000 & 100 & 3000 & & 2016 \\
\hline
\end{tabular}

a The value 20 is for the total of PFOA plus PFOS.

b The value 70 is for the total of PFOA plus PFOS.

c $\mathrm{AA}-\mathrm{EQS}=$ Annual Average - Environmental Quality Standards

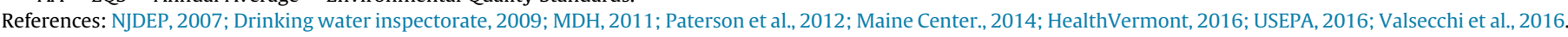

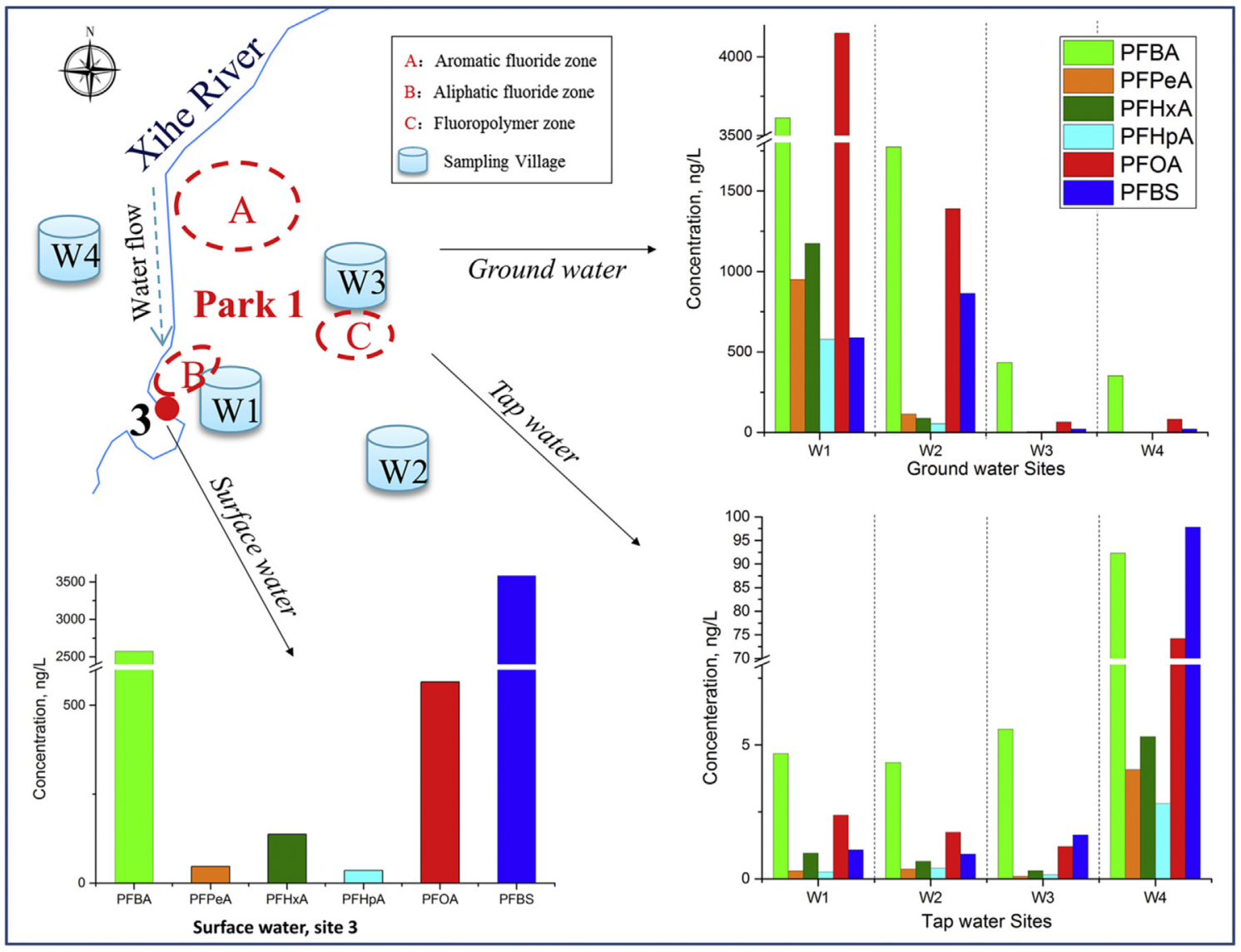

Fig. 6. Distribution of PFAAs levels among groundwater, tap water and river water nearby Park 1 in 2014. 
the highest mobility, and being the dominant presence in groundwater of all directions. PFBS were more dominant in river water than in groundwater. PFOA was dominant in the groundwater only in the nearest and downstream village of a specific production site. PFAAs levels in tap water were mostly below the criteria values except in one site (W4), which should be noticed by the tap water supplier and local government.

The fluorochemical industry is a complex source of PFAAs, as it generates numerous organic fluorine substances through various raw materials and processes. This study provided abundant information on the PFAA source identification, spatial and temporal trends, and different environmental behaviors of dominant PFAAs between surface water and groundwater. However, there are still some issues that need to be further studied in the future:

1) The possible presence in the discharges (and thereby in the rivers and groundwater) of PFAA precursors such as fluorotelomers (Fig. 5), which can modify the PFAA patterns in the different water bodies;

2) As a gathering of dozens of fluorochemical facilities, the removal efficiency of PFASs in the central WWTP needs to be evaluated to explore more effective approach for PFASs elimination;

3) With more facilities under development, higher PFAAs emission and potential health risks can be anticipated. Thus, the health risks of nearby residents need to be further investigated and managed.

\section{Acknowledgement}

This study was supported by the National Natural Science Foundation of China under Grant No. 414201040045 and No. 41371488, the International Scientific Cooperation Program with Grant No. 2012DFA91150, and the Key Project of the Chinese Academy of Sciences under Grant No.KZZD-EW-TZ-12. We would like to thank the editors and reviewers for their valuable comments and suggestions.

\section{Appendix A. Supplementary data}

Supplementary data related to this article can be found at http:// dx.doi.org/10.1016/j.envpol.2016.08.079.

\section{References}

3M, 2000. Phase-out Plan for PFOS-based Products. USEPA Docket. U.S. Environmental protection agency.

3M, 2002. Environmental, Health, Safety, and Regulatory (EHSR). Profile of Perfluorobutane Sulfonate (PFBS). http://solutions.3m.com/ 3MContentRetrievalAPI/BlobServlet?locale $=$ en

US\&lmd $=1120194514000 \&$ assetId $=1114270648708 \&$ assetType $=$ MMM Image\&blobAttribute $=$ ImageFile. July 22, 2016

$3 \mathrm{M}, 2008$. Frequently Asked Questions about PFBA. http://multimedia.3m.com/ mws/media/4379030/frequently-asked-questions-about-pfba.pdf. July 22 , 2016.

Ahrens, L., Bundschuh, M., 2014. Fate and effects of poly- and perfluoroalkyl substances in the aquatic environment: a review. Environ. Toxicol. Chem. 33 (9) 1921-1929.

Bao, J., Liu, W., Liu, L., Jin, Y., Dai, J., Ran, X., Zhang, Z., Tsuda, S., 2010. Perfluorinated compounds in the environment and the blood of residents living near fluorochemical plants in fuxin, China. Environ. Sci. Technol. 45 (19), 8075-8080.

Blum, A., Balan, S.A., Scheringer, M., Trier, X., Goldenman, G., Cousins, I.T. Diamond, M., Fletcher, T., Higgins, C., Lindeman, A.E., 2015. The Madrid statement on poly-and perfluoroalkyl substances (PFASs). Environ. health Perspect. 123 (5), A107-A111.

Buck, R.C., Franklin, J., Berger, U., Conder, J.M., Cousins, I.T., de Voogt, P., Jensen, A.A., Kannan, K., Mabury, S.A., van Leeuwen, S.P.J., 2011. Perfluoroalkyl and polyfluoroalkyl substances in the environment: terminology, classification, and origins. Integr. Environ. Assess. Manag. 7 (4), 513-541.

Davis, K.L., Aucoin, M.D., Larsen, B.S., Kaiser, M.A., Hartten, A.S., 2007. Transport of ammonium perfluorooctanoate in environmental media near a fluoropolymer manufacturing facility. Chemosphere 67 (10), 2011-2019.
DRINKING WATER INSPECTORATE, 2009. Guidance on the Water Supply (Water Quality) Regulations Specific to PFOS (Perfluorooctane Sulphonate) and PFOA (Perfluorooctanoic Acid) Concentrations in Drinking Water. http://dwi.defra. gov.uk/stakeholders/information-letters/2009/10_2009annex.pdf. July 22, 2016. European Commission, 2010. Analysis of the risks arising from the industrial use of Perfuorooctanoic acid (PFOA) and Ammonium Perfluorooctanoate (APFO) and from their use in consumer articles. Evaluation of the risk reduction measures for potential restrictions on the manufacture, placing on the market and use of PFOA and APFO. http://ec.europa.eu/enterprise/sectors/chemicals/files/docs studies/final_report_pfoa_pfos_en.pdf. July 22, 2016.

HealthVermont, 2016. Perfluorooctanoic Acid (PFOA) and Perfluorooctanesulfonic Acid (PFOS) Vermont Drinking Water Health Advisory. https://anrweb.vt.gov/ PubDocs/DEC/PFOA/PFOA - PFOS Health Advisories/Vermont/PFOA_PFOS HealthAdvisory_June_22_2016.pdf. July 22, 2016.

Holt, R., 2011. The influence of global regulatory changes and customer preferences on the development of alternatives to long chain fluorinated chemicals. www. oecd.org/ehs/pfc/46233887.ppt. July 22, 2016.

Liu, Z., Lu, Y., Wang, T., Wang, P., Li, Q., Johnson, A.C., Sarvajayakesavalu, S., Sweetman, A.J., 2016. Risk assessment and source identification of perfluoroalkyl acids in surface and ground water: spatial distribution around a mega-fluorochemical industrial park, China. Environ. Int. 91, 69-77.

Maine Center, 2014. Maximum Exposure Guideline for Perfluorooctanoic Acid in Drinking Water. http://www.maine.gov/dhhs/mecdc/environmental-health/ eohp/wells/documents/pfoameg.pdf. July 22, 2016.

$\mathrm{MDH}, 2007$. Health Based Values for Perfluorooctanoic (PFOA). http://www.health. state.mn.us/divs/eh/hazardous/topics/pfcs/pfoamemo0307.pdf. July 22, 2016.

MDH, 2011. Health-based Guidance for Water. http://www.health.state.mn.us/divs/ eh/risk/guidance/gw/table.html. July 22, 2016.

NJDEP, 2007. Guidance for PFOA in Drinking Water at Pennsgrove Water Supply Company. http://www.nj.gov/dep/watersupply/pdf/pfoa_dwguidance.pdf. July 22, 2016.

Oliaei, F., Kriens, D., Weber, R., Watson, A., 2013. PFOS and PFC releases and associated pollution from a PFC production plant in Minnesota (USA). Environ. Sci. Pollut. R. 20 (4), 1977-1992.

Paterson, L., Mitchell, I., Chatwell, I., Birk, R., 2012. Evaluation of Groundwater Transport of Perfluorinated Chemicals at a Former Fire-fighting Training Area. http://www.rpic-ibic.ca/documents/2012_fcs_presentations/Paterson_E.pdf. July 22, 2016.

Paul, A.G., Jones, K.C., Sweetman, A.J., 2009. A first global production, emission, and environmental inventory for perfluorooctane sulfonate. Environ. Sci. Technol. 43 (2), 386-392.

POPs Action in China, 2014. http://www.china-pops.org. March 19, 2015.

Post, G.B., Cohn, P.D., Cooper, K.R., 2012. Perfluorooctanoic acid (PFOA), an emerging drinking water contaminant: a critical review of recent literature. Environ. Res. 116, 93-117.

Saito, N., Harada, K., Inoue, K., Sasaki, K., Yoshinaga, T., Koizumi, A., 2004. Perfluorooctanoate and perfluorooctane sulfonate concentrations in surface water in Japan. J. Occup. Health 46 (1), 49-59.

Scheringer, M., Trier, X., Cousins, I.T., de Voogt, P., Fletcher, T., Wang, Z., Webster, T.F., 2014. Helsingør Statement on poly- and perfluorinated alkyl substances (PFASs). Chemosphere 114, 337-339.

Strynar, M., Dagnino, S., McMahen, R., Liang, S., Lindstrom, A., Andersen, E., McMillan, L., Thurman, M., Ferrer, I., Ball, C., 2015. Identification of novel perfluoroalkyl ether carboxylic acids (PFECAs) and sulfonic acids (PFESAs) in natural waters using accurate mass time-of-flight mass spectrometry (tofms). Environ. Sci. Technol. 49 (19), 11622-11630.

Taniyasu, S., Kannan, K., So, M.K., Gulkowska, A., Sinclair, E., Okazawa, T., Yamashita, N., 2005. Analysis of fluorotelomer alcohols, fluorotelorner acids, and short- and long-chain perfluorinated acids in water and biota. J. Chromatogr. A 1093 (1-2), 89-97.

Tsuda, T., Inoue, A., Igawa, T., Tanaka, K., 2010. Seasonal changes of PFOS and PFOA concentrations in lake biwa water. B Environ. Contam. Toxic. 85 (6), 593-597.

UNEP, 2009. The Conference Of The Parties 4 Of The Stockholm Convention (Cop-4) In Geneva Placed Perfluorooctane Sulfonate And Perfluorooctane Sulfonyl Fluoride (Pfos And Pfosf) In Annex B. http://chm.pops.int/Convention/ Pressrelease/COP4Geneva9May2009/tabid/542/language/en-US/Default.aspx. July 22, 2016.

USEPA, 2014. Emerging Contaminants-perfluorooctane Sulfonate (PFOS) and Perfluorooctanoic Acid (PFOA). http://www2.epa.gov/sites/production/files/201404/documents/factsheet_contaminant_pfos_pfoa_march2014.pdf. July 22, 2016.

USEPA, 2016. PFOA \& PFOS Drinking Water Health Advisories. https://www.epa. gov/ground-water-and-drinking-water/supporting-documents-drinkingwater-health-advisories-pfoa-and-pfos. July 22, 2016.

Valsecchi, S., Conti, D., Crebelli, R., Polesello, S., Rusconi, M., Mazzoni, M., Preziosi, E., Carere, M., Lucentini, L., Ferretti, E., Balzamo, S., Simeone, M.G., Aste, F., 2016. Deriving environmental quality standards for perfluorooctanoic acid (PFOA) and related short chain perfluorinated alkyl acids. J. Hazard. Mater. http:// dx.doi.org/10.1016/j.jhazmat.2016.04.055.

Wang, T.Y., Lu, Y.L., Chen, C.L., Naile, J.E., Khim, J.S., Park, J., Luo, W., Jiao, W.T., Hu, W.Y., Giesy, J.P., 2011. Perfluorinated compounds in estuarine and coastal areas of north Bohai Sea, China. Mar. Pollut. Bull. 62 (8), 1905-1914.

Wang, S., Huang, J., Yang, Y., Hui, Y., Ge, Y., Larssen, T., Yu, G., Deng, S., Wang, B., Harman, C., 2013. First report of a chinese PFOS alternative overlooked for 30 Years: its toxicity, persistence, and presence in the environment. Environ. Sci. Technol. 47 (18), 10163-10170. 
Wang, P., Lu, Y., Wang, T., Zhu, Z., Li, Q., Zhang, Y., Fu, Y., Xiao, Y., Giesy, J.P., 2015a. Transport of short-chain perfluoroalkyl acids from concentrated fluoropolymer facilities to the Daling River estuary, China. Environ. Sci. Pollut. Res. Int. 22 (13) 9626-9636.

Wang, Z., Cousins, I.T., Scheringer, M., Hungerbuehler, K., 2015b. Hazard assessment of fluorinated alternatives to long-chain perfluoroalkyl acids (PFAAs) and their precursors: status quo, ongoing challenges and possible solutions. Environ. Int. $75,172-179$.
Wang, P., Lu, Y., Wang, T., Meng, J., Li, Q., Zhu, Z., Sun, Y., Wang, R., Giesy, J.P., 2016 Shifts in production of perfluoroalkyl acids affect emissions and concentrations in the environment of the Xiaoqing River Basin, China. J. Hazard. Mater. 307, $55-63$.

Zhao, Z., Xie, Z., Tang, J., Sturm, R., Chen, Y., Zhang, G., Ebinghaus, R., 2015. Seasonal variations and spatial distributions of perfluoroalkyl substances in the rivers Elbe and lower Weser and the North Sea. Chemosphere 129, 118-125. 